\title{
Electrical Metrology Applications of LabVIEW Software
}

\author{
Hala M. Abdel Mageed, Ali M. El-Rifaie
}

National Institute for Standards, Giza, Egypt.

Email: halaabdelmegeed@yahoo.com, alisystem11@yahoo.com

Received December $21^{\text {st }}, 2012$; revised January $23^{\text {rd }}, 2013$; accepted January $31^{\text {st }}, 2013$

\begin{abstract}
Automation in measurement has wide range of electrical metrology applications and construction of powerful calibration software is one of the highly accurate metrological laboratories' priorities. Thus, two automatic systems for controlling and calibrating the electrical reference standards have been established at National Institute for Standards (NIS), Egypt. The first system has been built to calibrate the zener diode reference standards while the second one has been built to calibrate the electrical sourcing and measuring instruments. These two systems act as the comprehensive and reliable structure that, from the national electrical standards, disseminates the traceability to all the electrical units under calibration. The software of the two systems has been built using the Laboratory Virtual Instrument Engineering Workbench (LabVIEW) graphical language. The standard development procedures have been followed in the building of both systems software. The software requirement specifications as well as functional specifications are taken into consideration. Design, implementation and testing of the software have been performed. Furthermore, software validation for measurements' uncertainty as well as results' compatibility in both automatic and manual modes has been achieved.
\end{abstract}

Keywords: Electrical Metrology Applications; Automation; LabVIEW; Software Validation

\section{Introduction}

Programmable instruments are now widely employed and increasingly included in metrological laboratories, they can automatically perform high quality and time consuming operations required in calibration activity. Automation in measurement includes instruments controlling, measurement processing and results analysing. Accordingly, there is an effective request for software able to calibrate such instruments [1].

Available commercial software do not suit the needs of most metrological laboratories that work at higher levels of accuracy and precision; therefore, the need of software implementation arises at the first place in these laboratories. In addition, the importance of software validation becomes one of their main requirements due to the lack of comprehensive validation guidance materials [2].

In the National Metrology Institutes (NMIs), traceability for any automatic system is based on a set of reference standards linked to the primary standards [1]. In the fields of electrical and electronic standards, the parameters are voltage, current and resistance [3]. Consequently, it has been essential for NIS as the primary laboratory for DC electrical metrology in Egypt to construct a special programmable measurement system for the calibration of both NIS and customers' DC voltage reference standards.
This system has been built as the inclusive and consistent construction that, from the national standards, disseminates the traceability to all the DC voltage reference standards under calibration. NIS DC electrical laboratory has a Josephson Voltage Standard (JVS) as the primary standard for DC voltage and a group of zener diode reference standards as the DC voltage reference standard. This group includes two sets: the first set is the Fluke 734A and the second set is the Fluke 7000.

NIS electrical laboratory as well as high voltage laboratory have different types and models of sourcing instruments such as the Fluke 5720A, and the Wavetek 9100 reference calibrators; adding to measuring instruments such as Fluke 8508A, Fluke 8846A and HP 3458A digital multimeters (DMMs). Therefore, one more automatic calibration system using LabVIEW software has been built at NIS to control and calibrate the high sensitive electrical sourcing and measuring instruments.

This paper introduces two new automatic calibration systems that rely on Labview, Where both software and hardware of the systems are fully presented. The manual and automatic calibration results of calibrating $10 \mathrm{~V}$ outputs of seven Zener Diode units are introduced against the reference unit of the first set (Fluke 734A). In addition, a comparison between the manual and automatic calibration results of the sourcing and measuring instruments is introduced. 


\section{System Hardware}

The first system for calibrating zener diode reference standards consists of the NIS two zener diode reference standards sets; the first set is the Fluke 734A zener diode standard which includes four $732 \mathrm{~B}$ separate units and the second set is the Fluke 7000 voltage standard which includes four non separate units in one enclosure with four different output terminals. Adding to the 320A Data Proof low thermal scanner, 8.5 digit HP 3458A highly sensitive recently calibrated digital multimeter (DMM) and a PC controlled by General Purpose Interface Bus (GPIB/IEEE-488). One traceable zener diode of the first set (Fluke 732B with serial number, S.N. 8140009) which has been calibrated via our JVS is used as the reference unit (RU) to calibrate the other seven units of zener diodes as units under test (UUT1, UUT2 $\cdots$ UUT7).

Figure 1 illustrates the first system schematic diagram. The main operative instrument in this system is the low thermal scanner which used for scanning a number of instruments without physically changing the polarity of a device under test [4]. The Data Proof 320A (DP 320A) low thermal scanner with extremely low thermal offsets is ideal for automating precision measurements. It is re- motely controlled through the IEEE-488 interface [4]. This versatile dual scanner has 32 input channels and two pairs of output lines (A \& B) which makes it suitable for a wide variety of uses. Each unit of the first and the second sets has been connected to the scanner through shielded cables. The output lines A \& B of the DP 320A scanner have been connected to the High \& Low terminals of the HP 3458A DMM through the same type of shielded cables, where the Low terminals of output lines A \& B are shorted together. In order to calibrate the seven UUTs via the RU, the eight channels of the DP 320A scanner (from channel1 to channel8) have been used. Before using these eight channels in the calibration, they have been checked for their intended purpose. This check has been performed using the third configuration described in [5]. The reference unit and the seven under test Zener Diode units are listed in Table $\mathbf{1}$ with their models, their serial numbers and the scanner eight channels.

The second hardware is configured by the operator in relation with specific needs and several types of calibra tors and DMMs acting either as reference units or as units under calibration. Figure 2 illustrates the block

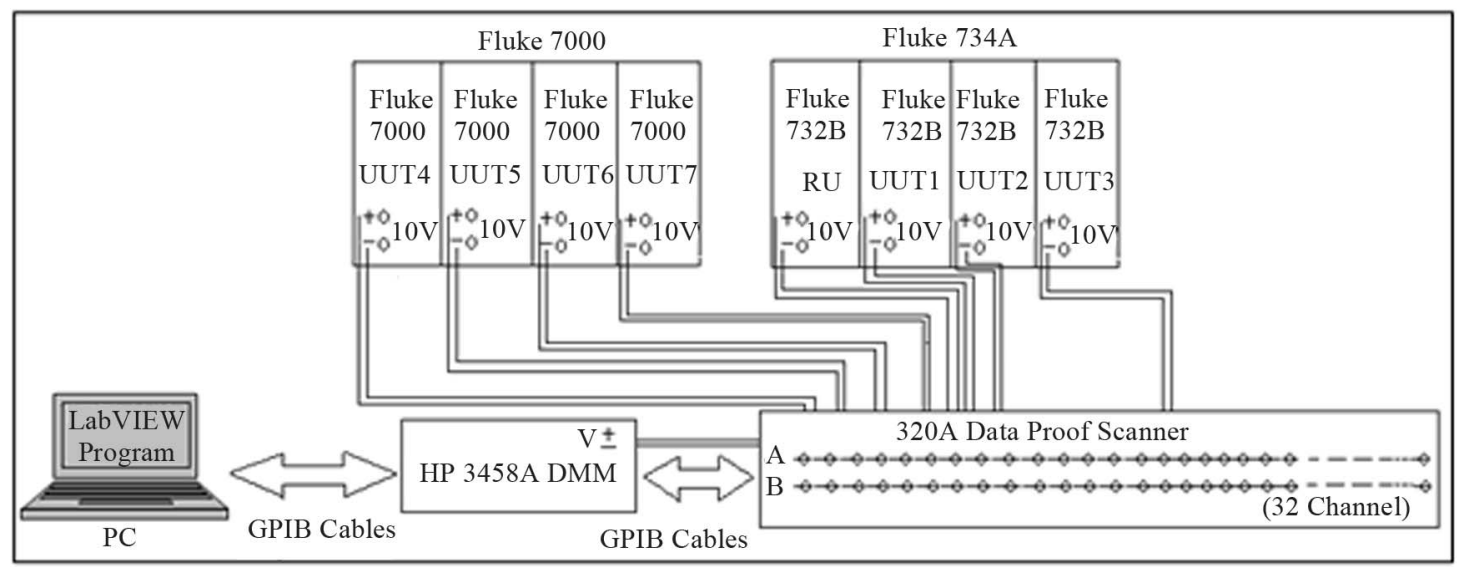

Figure 1. Schematic diagram of the zener diode reference standard automatic calibration system.

Table 1. Reference Unit and the seven under test units, their models, their serial numbers and the scanner eight channels.

\begin{tabular}{cccc}
\hline Zener Diode Units & Models & Serial Numbers & Scanner Channel Number (1 - 8) \\
\hline (RU) & Fluke 734B & 8140009 & 4 \\
(UUT1) & Fluke 734B & 8140006 & 1 \\
(UUT2) & Fluke 734B & 8140007 & 2 \\
(UUT3) & Fluke 734B & 8140008 & 3 \\
(UUT4) & Fluke 7000 & 45987 & 5 \\
(UUT5) & Fluke 7000 & 45988 & 6 \\
(UUT6) & Fluke 7000 & 41884 & 7 \\
(UUT7) & Fluke 7000 & 45989 & 8 \\
\hline
\end{tabular}




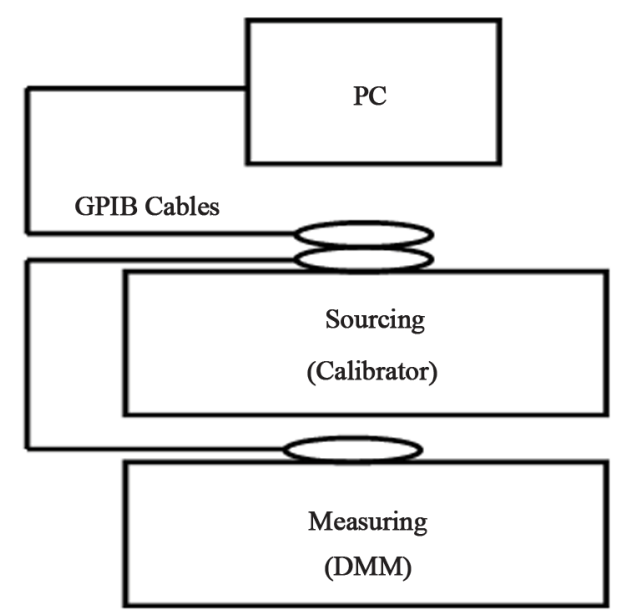

Figure 2. Block diagram of the automatic calibration system.

diagram of the implemented system, it consists of a programmable calibrator as a reference standard source for voltages, currents and resistances, a DMM as a reference standard measuring instrument, and a PC controlled by General Purpose Interface Bus (GPIB). The system is based on the PC equipped with a GPIB board which is used to automatically control the calibrator and to get the measurements of the DMM.

The temperature and relative humidity of the calibration laboratory were adjusted and fairly controlled to (23 $\pm 1)^{\circ} \mathrm{C}$ and $(50 \% \pm 10 \%)$ respectively.

\section{System Software}

The zener diode reference standards calibration system software as well as the sourcing and measuring electrical instruments have been built using LabVIEW graphical language. This software is constructed to control the calibration system, eliminate the operator's errors, allow statistical proceeding of the results in rather short time and generate a complete visual statistical information report from the performed calibration. The standard procedures for development of software use the V-Model for software life-cycle. This V-Model includes the software requirements specification, functional specification, design specification, testing, and implementation [6]. The development principle of V-Model shown in Figure 3 has been followed in the building of the calibration system software. Preparing the requirements' specifications is a significant part of the software life-cycle development. The function specification is more comprehensive than the requirements specification and must include full explanation of each function. After problem identification and requirements declaration, the software can be designed. In the implementation step, the code is created regarding the agreed design of the software. After performing module and integration testing the operational testing is finally performed to fulfill the development

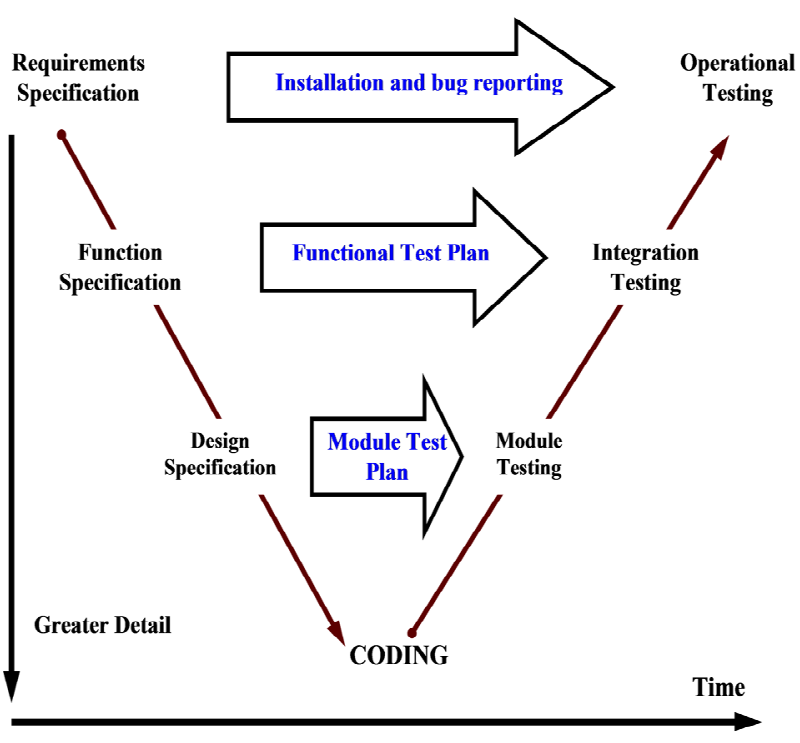

Figure 3. V-Model for software life-cycle.

principle of the V-model software life-cycle. The following procedures will clearly explain this development principle.

\subsection{Requirements Specification}

In this part, the description of the problem and the approach which will be followed to solve this problem is recognized. The Requirements Specification is the input to the software design process. Here, the problem which has to be solved by the software development is the performing of the automatic calibration systems of the zener diode reference standards as well as electrical sourcing and measuring instruments. The followed approach is outlining the main functions which have to be carried out. The first software functions are:

- Preliminary warming-up of the calibration systems.

- Controlling the scanner to choose the specified UUT which will be calibrated by the RU in sequence required by their calibration procedures (in forward and reverse directions).

- Controlling the digital multimeter to read the difference between the output voltages of the RU and the specified UUT in both directions.

- Collecting the measured data indicated by the digital multimeter.

- Performing calculations based on the measured data regarding the formulas imposed by the calibration procedures.

- Generating the measurements report and graphical visualization of the outputs.

In the same manner, the second software functions are warming up of the calibration system, controlling the calibrator's output, adjusting the DMM to read, collecting the DMM measured data, and generating the final 
measurements report.

\subsection{Functions Specification}

Functions specification illustrates how each requirement is to be met. Adding together, it should emphasize any inconsistency if complete agreement is not realistic. The functional specification covers [6,7]:

- Software and hardware environment.

- Description of the software's functions.

- Input and the output data.

- Operator's interface to the system.

- Special restrictions that will be applied to the system.

- Software management.

Finally, the function specifications will cover all the details of how the end-user of the automatic system is to interact with this system. Eventually, each function described will require a test to prove compliance with the specification. Therefore, this document will be used as the input to the functional test.

\subsection{Software Design}

The software design is a record of how the requirements will be implemented. The design document may use state diagrams, flowcharts or formal methods to describe the software design. In this case, LabVIEW is used as the development language. It is a rapid development system and provides useful documentation tools that can be used in the development of the design document. The design document includes the program structure on the base of LabVIEW Virtual Instruments (VI) hierarchy, module (VI) design, LabVIEW coding conventions and LabVIEW tools [8]. Each module defined in this design will require a test to verify compliance with the requirements.

\subsection{Implementation and Testing}

When the software design is finished the software can be fully implemented. The code is created concerning decided design of the software. LabVIEW is a graphical programming language and states transition diagram to follow the coding conversion declared in the design. The module test is executed to prove that each module (VI) requirement in the design document is fulfilled. The excellent test will illustrate that the module achieves the designed function. The functional test is performed as well. This test level is higher than the level of the module test. It tests how the modules work together concerning functions defined in the functional specification. Moreover, it tests the operator interfaces and output results for stability and accuracy. After module and functional successfully testing, the operational testing is performed by implementing the LabVIEW software in the calibration system. It is preferred to perform the functional tests once more in the final version of the LabVIEW software.

\subsection{LabVIEW Front Panel and Block Diagram}

NIS LabVIEW software for calibrating zener diode reference standards as well as electrical sourcing and measuring instruments have been built following the application of the previous procedures. LabVIEW consists of two main components: the front panel and the block diagram; besides, it also contains a comprehensive library for data collection, analysis, presentation and storage. Program execution is determined by the structure of a graphical block diagram on which the programmer connects different function nodes by drawing wires. These wires propagate variables and any node can execute as soon as all its input data become available. The front panel is used to interact with the user when the program is running. User can control the program, change inputs, and see data updated in real time $[9,10]$. The front panel and the block diagram of the automatic calibration systems have been carried out. Uncertainty of measurement is the doubt that exists about the result of any measurement. For every measurement-even the most careful - there is always a margin of doubt. International metrology organizations recommend that uncertainties should have two types, "Type A" and "Type B", based on the method by which they are evaluated [11]. Where, "Type A" is the uncertainty using statistics (usually from repeated readings). While, "Type B" evaluation is the uncertainty estimate from any other information. The combined uncertainty equals to the root sum square of all the uncertainty contributions. The expanded uncertainty is obtained by multiplying the combined uncertainty by coverage factor " $\mathrm{k}$ ". The value of coverage factor gives the confidence level for the expanded uncertainty. Most commonly, the overall uncertainty is scaled by using the coverage factor $\mathrm{k}=2$, to give a level of confidence of approximately 95\% [11,12]. The new automatic calibration systems have the facility to automatically calculate the measurements repeatability, store the data, record, and report the calibration results. Thirty readings have been automatically taken and transferred to the excel sheets by the software. For example, Figure 4 illustrates the automatic zener diode reference standards calibration system front panel. It includes the exact time and date of performing the calibration adding to the different (Type B) uncertainty components which contribute in the uncertainty budget. The uncertainty of the calibration measurements has been estimated based on the ISO/IEC 17025 [12] and the expanded uncertainty has been reported by the software.

\section{Results and Software Validation}

The two automatic systems have been constructed for calibrating the Zener Diode Reference Standards (1.018 $\mathrm{V}$ and $10 \mathrm{~V}$ outputs), as well as the electrical sourcing 


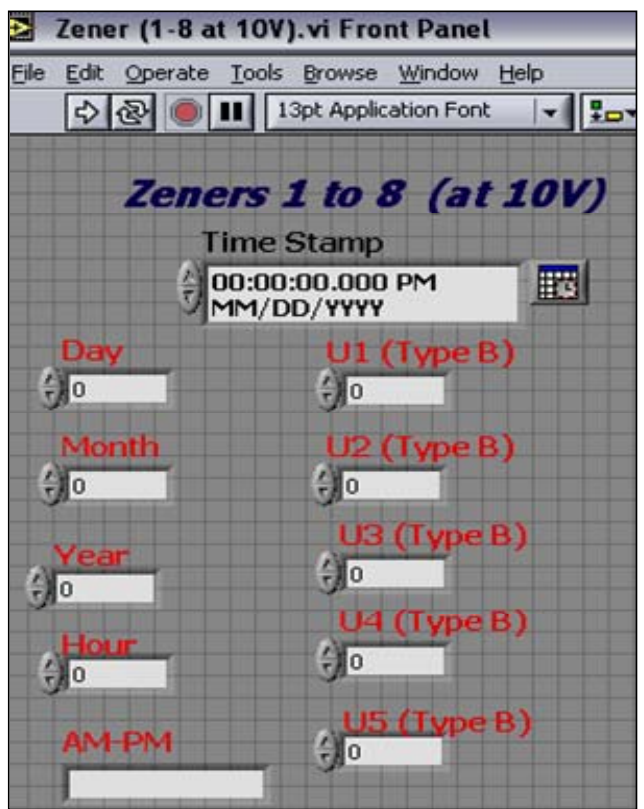

Figure 4. Front Panel of the zener diode reference standard automatic calibration system.

and measuring instruments. By functionality testing of the systems hardware and software, it is fairly demonstrated that, the automatic calibration procedures execu- tion is more rapidly than the manual procedures performing. What is more, in the automatic mode, the operator full attendance is not needed as in the manual mode and a better calibration procedure is guaranteed. In addition, to the system functionality test and the importance of achieving the measurements reliability guarantee in metrology science [13], the two systems have been validated.

\subsection{Software Validation of First System}

In order to validate the first system for its specific purpose, it has been utilized in calibrating the NIS Zener Diode Reference Standards Group at $10 \mathrm{~V}$ output and the results have been compared with those obtained by their manual calibration. Then, the calibration measurements uncertainty in both modes has been evaluated and compared by (automatically and manually) testing the system under the same calibration conditions ten times, in five consecutive days. Each day ten observations have been taken to evaluate the average value. The same scanner, DMM, connecting cables and the same environmental conditions of the lab have been used in both manual and automatic calibrations. The repeatability evaluated as a $1 \sigma$ type (A) uncertainty and in between days variance in voltages has been considered. Figures 5 and $\mathbf{6}$ show the

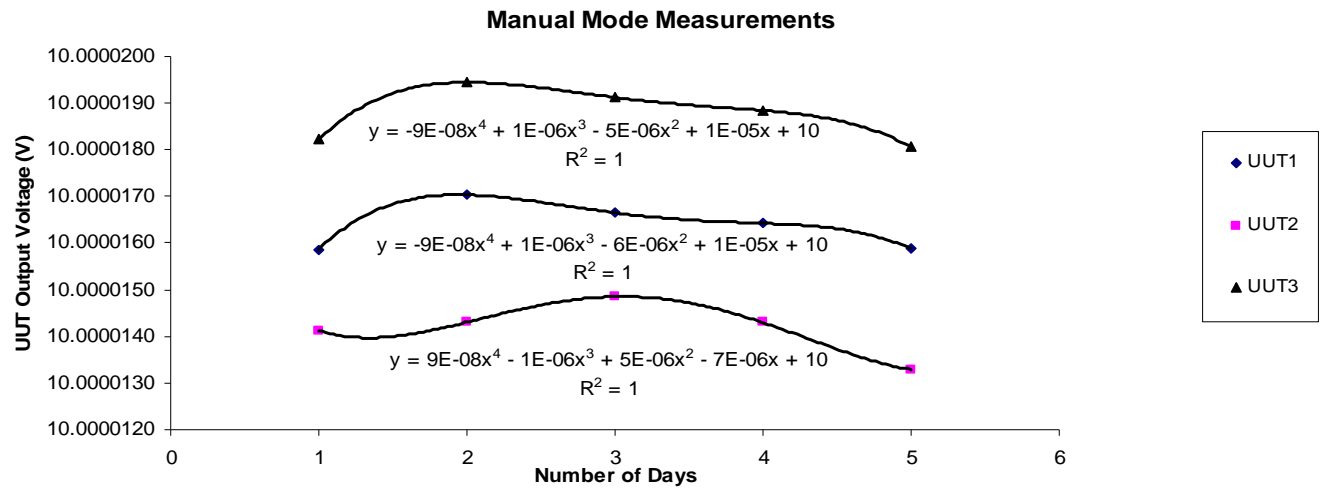

Figure 5. Five days manual output voltage of the UUT1, UUT2 and UUT3.

Automatic Mode Measurements
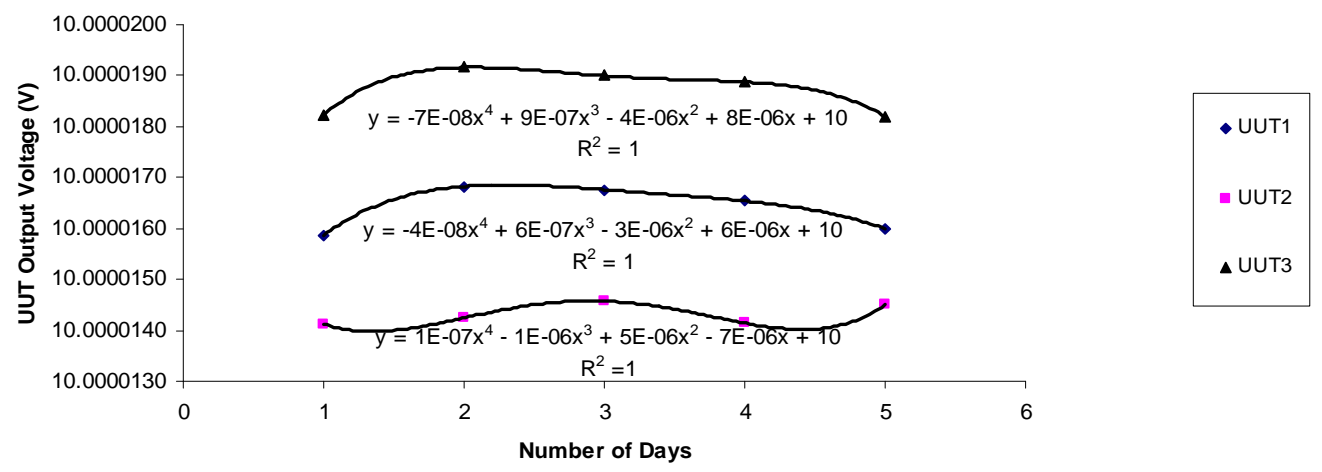

Figure 6. Five days automatic output voltage of the UUT1, UUT2 and UUT3. 
five days data of the UUT1, UUT2 and UUT3 in the manual mode and automatic mode respectively. Table 2 presents the estimated uncertainty budget of UUT1 calibration results while, Table 3 illustrates the automatic and manual $10 \mathrm{~V}$ output calibration results with their expanded uncertainty values (at 95\% confidence level, $\mathrm{k}=2$ ).

\subsection{Software Validation of the Second System}

The second automatic system has been built for calibrating NIS as well as customers' high sensitive calibrators and DMMs. To validate the programmable calibration system for its specific purpose, the Fluke 5720A, Wavetek 9100 calibrators, HP 3458A, and Fluke 8508A, 8846A DMMs have been calibrated in both manual and automatic modes for all their functions (voltage, current, resistance) and all their ranges. All automatically obtained results have been compared with those manually obtained. The calibration measurements repeatability in both modes are then evaluated and compared by testing the system under the same environmental conditions (both automatically and manually. The repeatability evaluated as a $1 \sigma$ type (A) uncertainty [14].
Table 4 shows samples of the manual and automatic calibration results for different electrical sourcing and measuring instruments. Samples of these results are illustrated from Figures 7-11.

As shown in Figures 5 and 6, the drift rates of UUT1, UUT2, UUT3 average outputs have been calculated manually and automatically using quadratic polynomial fit. The drift rates of average outputs in the automatic mode are better than the manual ones. In Table $\mathbf{3}$, it is clearly shown that the automatic calibration results for the $10 \mathrm{~V}$ outputs of the under test seven Zener Diode units are very close to their corresponding manual results. Furthermore, it is quite verified that, the evaluated uncertainty values in automatic mode are smaller than the values manually obtained. On the other hand, Table 4 and Figures (7)-(11) clearly show that the automatic results of the second system are very close to their corresponding manual results. Moreover, their evaluated repeatability values in automatic mode are smaller than the values manually obtained. As a result, a full compatibility and an improved repeatability have been achieved with measurements made by the first and the second automatic system.

Table 2. Uncertainty budget of UUTs calibration results.

\begin{tabular}{|c|c|c|c|}
\hline Sources of Uncertainty & Type of Uncertainty & \multicolumn{2}{|c|}{ Combined Uncertainty } \\
\hline $\begin{array}{l}\text { RU uncertainty from its } \\
\text { calibration certificate (U1) }\end{array}$ & Type B & \multicolumn{2}{|c|}{$1.00 \mathrm{E}-07$} \\
\hline Linearity deviation of DMM (U2) & Type B & \multicolumn{2}{|c|}{$0.058 \mathrm{E}-6$} \\
\hline Temperature deviation of RU Zener (U3) & Type B & \multicolumn{2}{|c|}{$0.023 \mathrm{E}-06$} \\
\hline Temperature deviation of UUT Zener (U4) & Type B & \multicolumn{2}{|c|}{$0.046 \mathrm{E}-06$} \\
\hline Drift in RU value (U5) & Type B & \multicolumn{2}{|c|}{$0.058 \mathrm{E}-6$} \\
\hline Thermal emf in calibrating UUT (U6) & Type B & \multicolumn{2}{|c|}{$0.058 \mathrm{E}-6$} \\
\hline Repeatability (U7) & Type A & $\begin{array}{c}\text { Auto } \\
4.32 \mathrm{E}-08\end{array}$ & $\begin{array}{c}\text { Manual } \\
6.41 \mathrm{E}-08\end{array}$ \\
\hline Reproducibility (U8) & Type A & Auto & Manual \\
\hline
\end{tabular}

Table 3. Automatic \& manual $10 \mathrm{~V}$ output calibration results with their expanded uncertainty.

\begin{tabular}{ccc}
\hline Zener & $\begin{array}{c}\text { Automatic Calibration Results } \\
\text { of 10 V Output }(\mathrm{V})\end{array}$ & $\begin{array}{c}\text { Manual Calibration Results } \\
\text { of 10 V Output }(\mathrm{V})\end{array}$ \\
\hline UUT1 & $10.0000164 \pm 0.50 \mathrm{E}-6$ & $10.0000164 \pm 0.56 \mathrm{E}-6$ \\
UUT2 & $10.0000143 \pm 0.36 \mathrm{E}-6$ & $10.0000142 \pm 0.61 \mathrm{E}-6$ \\
UUT3 & $10.0000187 \pm 0.51 \mathrm{E}-6$ & $10.0000187 \pm 0.62 \mathrm{E}-6$ \\
UUT4 & $09.9999479 \pm 0.81 \mathrm{E}-6$ & $09.9999450 \pm 1.72 \mathrm{E}-6$ \\
UUT5 & $09.9999557 \pm 0.61 \mathrm{E}-6$ & $09.9999557 \pm 0.62 \mathrm{E}-6$ \\
UUT6 & $10.0000218 \pm 0.46 \mathrm{E}-6$ & $10.0000222 \pm 1.88 \mathrm{E}-6$ \\
UUT7 & $09.9999529 \pm 0.63 \mathrm{E}-6$ & $09.9999529 \pm 0.66 \mathrm{E}-6$ \\
\hline
\end{tabular}


Table 4. Samples of the automatic and manual calibration results for different electrical sourcing and measuring instruments.

\begin{tabular}{|c|c|c|c|c|c|}
\hline \multirow{2}{*}{ Electrical Instrument } & \multirow{2}{*}{ Ranges } & \multicolumn{2}{|c|}{ Manual mode } & \multicolumn{2}{|c|}{ Automatic mode } \\
\hline & & Value & Type A & Value & Type A \\
\hline \multirow{4}{*}{$\begin{array}{c}\text { Fluke 5720a } \\
\text { Calibrator @ } \\
\text { Fluke 8508A DMM }\end{array}$} & AC Volt1000V@50 Hz & 999.90010 & $5.27 \mathrm{E}-3$ & 999.90260 & $2.69 \mathrm{E}-3$ \\
\hline & DC Current 2 A & 1.9998098 & $2.07 \mathrm{E}-6$ & 1.9997699 & $1.64 \mathrm{E}-6$ \\
\hline & AC Current1A@50 Hz & 0.9971814 & $4.23 \mathrm{E}-4$ & 0.9997919 & $2.13 \mathrm{E}-5$ \\
\hline & Resistance $1 \mathrm{~K} \Omega$ & 1.0016791 & $4.48 \mathrm{E}-4$ & 1.0016599 & $9.41 \mathrm{E}-5$ \\
\hline \multirow{4}{*}{$\begin{array}{c}\text { Fluke 5720a } \\
\text { Calibrator @ } \\
\text { Fluke 8846A DMM }\end{array}$} & DC Volt $100 \mathrm{~V}$ & 99.999329 & $8.54 \mathrm{E}-6$ & 99.999506 & $5.71 \mathrm{E}-6$ \\
\hline & AC Volt100 V@1kHz & 99.897468 & $5.25 \mathrm{E}-5$ & 99.896279 & $4.72 \mathrm{E}-5$ \\
\hline & AC Current1 A@1kHz & 1.0000061 & $6.60 \mathrm{E}-06$ & 1.0000855 & $7.98 \mathrm{E}-07$ \\
\hline & Resistance $100 \Omega$ & 99.993945 & $6.61 \mathrm{E}-05$ & 99.973403 & $5.28 \mathrm{E}-05$ \\
\hline \multirow{5}{*}{$\begin{array}{c}\text { Wavetek } 9100 \\
\text { Calibrator @ } \\
\text { Hp 3458A DMM }\end{array}$} & DC Volt $10 \mathrm{~V}$ & 10.000008 & $7.48 \mathrm{E}-06$ & 10.000002 & $4.83 \mathrm{E}-06$ \\
\hline & AC Volt10V@1kHz & 9.9893166 & $8.04 \mathrm{E}-06$ & 9.9893268 & $4.22 \mathrm{E}-06$ \\
\hline & DC Current $10 \mathrm{~mA}$ & 9.9969120 & $4.93 \mathrm{E}-08$ & 9.9971854 & $2.22 \mathrm{E}-08$ \\
\hline & AC Current 100 mA@50 Hz & 99.991467 & $9.17 \mathrm{E}-07$ & 99.991469 & $7.23 \mathrm{E}-07$ \\
\hline & Resistance $100 \Omega$ & 99.993945 & $6.61 \mathrm{E}-05$ & 99.993403 & $5.28 \mathrm{E}-05$ \\
\hline
\end{tabular}

Manual and Automatic Values of $10 \mathrm{~V}-\mathrm{DC}$

Automatic Mode

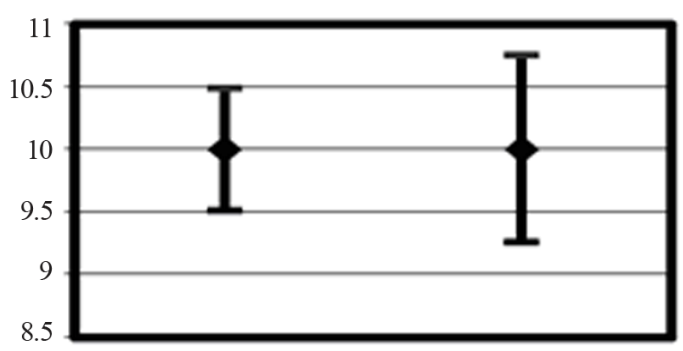

Figure 7. Manual and automatic values of $10 \mathrm{~V}$-DC.

Manual and Automatic Values of $1000 \mathrm{V@}$,50Hz-AC

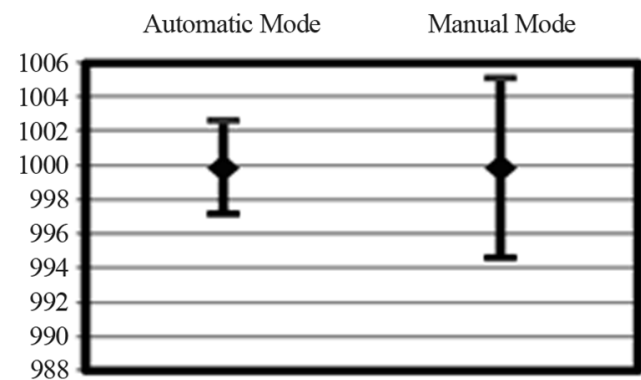

Figure 8. Manual and automatic values of 1000 V@50 Hz-AC.
Manual and Automatic Values of 2A-DC Current

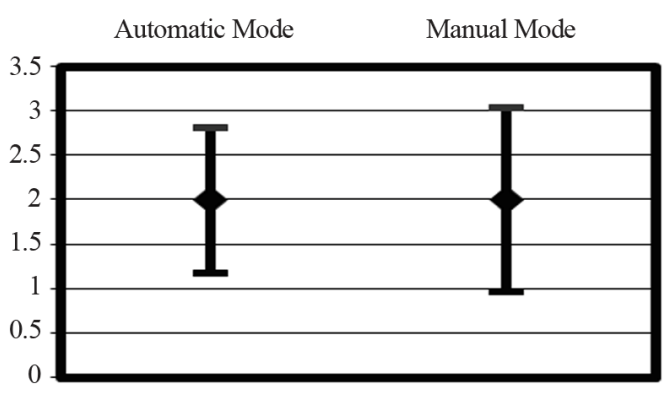

Figure 9. Manual and automatic values of 2 A-DC.

Manual and Automatic Values of $100 \mathrm{~mA} @ 50 \mathrm{~Hz}-\mathrm{AC}$ Current

Automatic Mode

Manual Mode

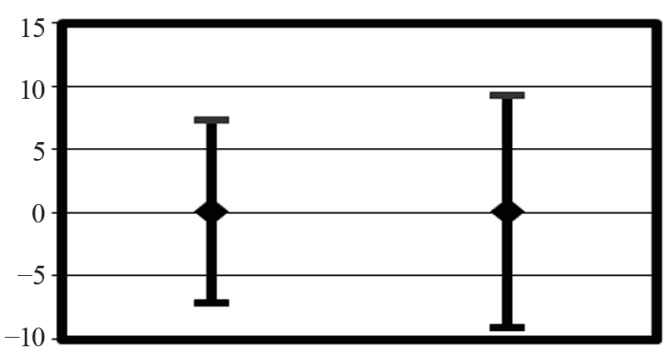

Figure 10. Manual and automatic values of $100 \mathrm{~mA} @ 50$ Hz-AC. 
Manual and Automatic Values of 100 OHMR Resistance

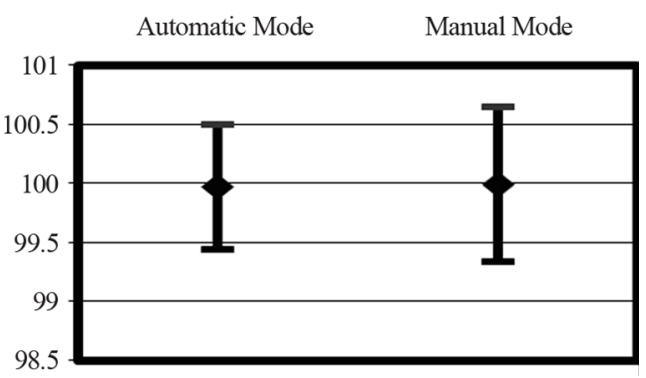

Figure 11. Manual and automatic values of $100 \Omega$ resistance.

\section{Conclusion}

Two automatic calibration systems using LabVIEW graphical language have been built at NIS. The first one is performed for calibrating DC Zener Diode reference standards while the second one is implemented for calibrating the electrical sourcing and measuring instruments. Both systems have been designed and implemented as consistent construction from the national electrical reference standards that can disseminate the traceability of all the instruments under calibration. The new automatic systems enable fast and reliable measurement function including statistical proceeding, measurement results visualization on the screen and generation of measurement report. This significantly improves the measurement process as well as the calibration process. The software validation process for the new automated systems has shown the functionality of the two systems and a full compatibility with calibration measurements made manually and by the automated systems. Adding to, performing rather enhanced uncertainty results in the automatic operation. The new automatic calibration systems are now in use for the calibrations of both the NIS and the customer DC Zener Diode reference standards as well as the electrical sourcing and measuring instruments.

\section{REFERENCES}

[1] U. Poliano, G. C. Bosco and M. Lanzillotti, "Generalized Automatic System for AC/DC Transfer AC Voltage and
AC Current Measurements," IEEE Transactions on Instrumentation and Measurement, Vol. 55, No. 5, 2006, pp. 1747-1751. doi:10.1109/TIM.2006.880958

[2] R. Flegar and T. Tasic, "Software Validation in Measurement \& Testing," INCOLAB Conference, Prague, 4 December 2003.

[3] S. K. Sharma and V. N. Ojha, "Performance Evaluation and Software Validation of Automatic Bank of DC Reference Standard," Journal of Scientific \& Industrial Research, Vol. 64, No. 7, 2005, pp. 487-490.

[4] http://www.nist.gov/manuscriptpublicationsearch.cfm?pu b_id=32563

[5] S. K. Jaiswal, "Complete Characterization of a Low Thermal Scanner for Automatic Voltage Measurement," $M A$ PAN-Journal of Metrology Society of India, Vol. 23, No. 1, 2008, pp. 31-38

[6] J. Mountford and G. I. Parkin, "Best Practice in Software Development - A Case Study in LabVIEW Illustrated by the UHTBB Safety System Monitor Project," NPL Report DEM-ES, Teddington, 2007.

[7] I. Hadzhieva-Borisova, "Software for Automated AC-DC Measurement System," International Scientific Conference Computer Science, Sofia, 2008, pp. 528-532

[8] National Instruments, "LabVIEW Development Guidelines," 2003.

[9] National Instruments, "Introduction to LabVIEW," Part No. 323668B-01, 2003.

[10] National Instruments, "LabVIEW Quick Start Guid," Part No. 321527B-01, 1998.

[11] W. A. Everett, "Calibration: Philosophy in Practice," 2nd Edition, Fluke Corporation, Everett, 1994.

[12] United Kingdom Accreditation Service, "The Expression of Uncertainty and Confidence in Measurement," 2007.

[13] C. Capua, D. Grillo and E. Romeo, "The Implementation of an Automatic Measurement Station of the Determination of the Calibration Intervals for a DMM," IEEE International Conference on Virtual Environments, HumanComputer Interfaces and Measurement Systems, Spain, 10-12 July 2006, pp. 58-62.

[14] Saudi Arabian Standards Organization (SASO), "Guide to the Expression of Uncertainty in Measurement," 2006. 